OPEN ACCESS

Edited by:

Ilkwon Oh,

Korea Advanced Institute of Science \&

Technology (KAIST), South Korea

Reviewed by:

Erin Krista Cressman

University of Ottawa, Canada

Donato Romano,

Institute of BioRobotics, Sant'Anna

School of Advanced Studies, Italy

*Correspondence:

Giacomo Severini

giacomo.severini@ucd.ie

Specialty section:

This article was submitted to

Bionics and Biomimetics,

a section of the journal

Frontiers in Bioengineering and

Biotechnology

Received: 21 August 2019 Accepted: 22 November 2019 Published: 06 December 2019

Citation: Castronovo AM, Giles Doran C, Holden M and Severini G (2019) Analysis of the Effectiveness of

Sub-sensory Electrical Noise Stimulation During Visuomotor Adaptations in Different Visual Feedback Conditions. Front. Bioeng. Biotechnol. 7:399.

doi: 10.3389/fbioe.2019.00399

\section{Analysis of the Effectiveness of Sub-sensory Electrical Noise Stimulation During Visuomotor Adaptations in Different Visual Feedback Conditions}

\author{
Anna Margherita Castronovo ${ }^{1}$, Ciara Giles Doran ${ }^{1}$, Méabh Holden ${ }^{1}$ and \\ Giacomo Severini ${ }^{1,2,3 *}$
}

${ }^{1}$ School of Electrical and Electronic Engineering, University College Dublin, Dublin, Ireland, ${ }^{2}$ UCD Centre for Biomedical Engineering, University College Dublin, Dublin, Ireland, ${ }^{3}$ Insight Centre for Data Analytics, University College Dublin, Dublin, Ireland

Sub-sensory electrical noise stimulation has been shown to improve motor performance in tasks that mainly rely on proprioceptive feedback. During the execution of movements such as reaching, proprioceptive feedback combines dynamically with visual feedback. It is still unclear whether boosting proprioceptive information in tasks where proprioception mixes with vision can influence motor performance. To better understand this point, we tested the effect of electrical noise stimulation applied superficially to the muscle spindles during four different experiments consisting of isometric reaching tasks under different visual feedback conditions. The first experiment $(n=40)$ consisted of a reachand-hold task where subjects had to hold a cursor on a target for $30 \mathrm{~s}$ and had visual feedback removed $10 \mathrm{~s}$ into the task. Subjects performed 30 repetitions of this task with different stimulation levels, including no stimulation. We observed that trials in which the stimulation was present displayed smaller movement variability. Moreover, we observed a positive correlation between the level of stimulation and task performance. The other three experiments consisted of three versions of an isometric visuomotor adaptation task where subjects were asked to reach to random targets in $<1.5 \mathrm{~s}$ (otherwise incurring in negative feedback) while overcoming a $45^{\circ}$ clockwise rotation in the mapping between the force exerted and the movement of the cursor. The three experiments differed in the visual feedback presented to the subjects, with one group $(n=20)$ performing the experiment with full visual feedback, one $(n=10)$ with visual feedback restricted only to the beginning of the trajectory, and one $(n=10)$ without visual feedback of the trajectory. All subjects performed their experiment twice, with and without stimulation. We did not observe substantial effects of the stimulation when visual feedback was present (either completely or partially). We observed a limited effect of the stimulation in the absence of visual feedback consisting in a significant smaller number of negative-feedback trials 
and a significant smaller movement time in the first block of the adaptation phase. Our results suggest that sub-sensory stimulation can be beneficial when proprioception is the main feedback modality but mostly ineffective in tasks where visual feedback is actively employed.

Keywords: proprioception, visual feedback, stochastic resonance, visuomotor adaptation, motor control

\section{INTRODUCTION}

Mechanical and electrical noise stimulation targeting joints and muscles can alter the kinesthetic sense and lead to improved motor performances (Cordo et al., 1996; Gravelle et al., 2002; Priplata et al., 2002, 2006; Collins et al., 2003, 2014; Ross and Guskiewicz, 2006; Mendez-Balbuena et al., 2012; Iliopoulos et al., 2014; Miranda et al., 2016; Severini and Delahunt, 2018). Mechanical noise stimulation directly modifies the response of sensory receptors, while electrical noise stimulation alters the baseline transmembrane potential of the stimulated afferents making them more likely to fire in response to a weak stimulus (Gravelle et al., 2002; Miranda et al., 2016). Both effects are supposedly related to stochastic resonance (SR), a phenomenon for which noise can improve the reception of weak signals in threshold-based systems (Gammaitoni, 1995). By the SR phenomenon, noise added to the input of a threshold-based receiving system can improve the detection of a weak input signal by spuriously amplifying it. Values of noise that are too low may not bring the weak signal above the receiving threshold, while values of noise that are too high may mask the characteristics of the input signal and lead to erroneous detections. Therefore, the SR phenomenon predicts the presence of an optimal level of stimulation that maximizes the performance of the receiving system.

The SR phenomenon has been observed to occur in response to noise stimulation in biological systems in general (Collins et al., 1995), and in human sensory receptors in particular (Cordo et al., 1996; Mendez-Balbuena et al., 2012, 2015; Iliopoulos et al., 2014). Proprioception plays a crucial role during the execution and learning of voluntary movements (Fleishman and Rich, 1963; Sober and Sabes, 2003) and sensory deficits have been shown to affect motor re-learning after a neurological injury (Vidoni and Boyd, 2009). Several studies have shown that superficial electrical noise stimulation targeting sensory receptors at sub-sensorial current levels (intended as current levels that do not elicit conscious perception) can improve performance during different motor tasks in healthy subjects (Magalhaes and Kohn, 2012, 2014; Iliopoulos et al., 2014), elderlies (Gravelle et al., 2002), and individuals suffering from sensory loss (Collins et al., 2014). In all these experiments, the motor tasks selected (i.e., single leg stance) relied heavily on proprioception as sensory feedback modality. Recently, we were also able to show that, in opposition to the results obtained using sub-sensorial stimulation, supra-sensorial currents lead to a decrease in motor performance during mildly challenging balance tasks (Severini and Delahunt, 2018), although it is not clear whether this effect is caused by a reaction to the conscious sensation of the stimulation or by the degradation in performance expected by the SR model for levels of noise above the optimal one. It has been proposed that sub-sensory noise stimulation could be used to improve the quality and quantity of available proprioceptive information during rehabilitation of patients affected by proprioceptive deficits (Collins et al., 2003). In this scenario, since motor learning in rehabilitation is often associated with complex tasks (e.g., walking and reaching) where several sensory feedback modalities are integrated and employed at the same time, it is paramount to understand what could be the effect of boosting proprioception when several feedback modalities are available. This latter point is still unexplored in literature. In fact, while most studies employing sub-sensory stimulation have shown its benefits in tasks where proprioception is the main feedback modality, it is not clear what its effect would be in tasks where proprioception integrates (or competes) with other sensory modalities, such as vision. As a case in point, during reaching movements proprioceptive and visual feedback (VF) are weighted flexibly depending on the task and on the quality and availability of feedback (Sober and Sabes, 2003, 2005). In this perspective, externally altering the natural "gain" of proprioception through sub-sensorial stimulation could affect the sensory weighting that happens during the task and impact motor performance. It cannot be excluded also that the weighting process could completely "bypass" the artificial sensory boost.

In this work we aim at testing if enhancing proprioception through sub-sensorial electrical stimulation can alter motor performance during reach-and-hold and visuomotor adaptations (VMA) tasks under different VF conditions. The VMA task was selected over a standard reaching task to probe potential effects of enhancing proprioception during challenging exercises requiring a motor re-calibration.

Moreover, as motor adaptation is considered one of the processes constituting motor learning (Shadmehr and Wise, 2005; Krakauer, 2009), our experiments aim also at providing additional information on the usability of SR stimulation as an additional aid during rehabilitation therapy of reaching movements. In our experiments, we asked subjects to perform a reach-and-hold task where VF was removed during the hold part of the task. Subjects repeated the task several times with different levels of sub-sensorial stimulation applied to the muscles driving the movement. This experiment was designed for determining the subject-specific optimal stimulation level, defined as the current level minimizing movement variability during the hold phase of the movement when VF was not present (thus in the portion of the task that was only reliant on proprioceptive feedback). Subjects were then split in three groups and each group performed a version of a VMA experiment twice, once 
with optimal sub-sensory stimulation (Stim condition) and once with no stimulation (NoStim condition), in a random order. One group performed the experiment with the VF always present (Full VF), one with VF limited to the initial part of the reaching movement (Limited VF) and one with VF only of the starting positions and end results of each movement (No VF). These three VF conditions were selected to examine the impact of enhancing proprioception in both the planning and on-line adjustment phases of the movement. We report here a limited effect of subsensory stimulation on task performance only when the VF is not present. These findings have major implications for evaluating the use of sub-sensory electrical stimulation during the execution of complex tasks.

\section{METHODS}

\section{Participants}

A total of 40 healthy individuals (19 females, age $24.0 \pm$ 4.3 years) volunteered for this study by signing an informed consent. All the experimental procedures were approved by the Ethical Committee of University College Dublin and have been conducted according to the WMA's declaration of Helsinki. No personal or sensitive data were collected for the study. The study consisted of four different experiments executed using the same experimental setup (Figure 1). Each subject participated to two of the four experiments. One of the two experiments, the Optimal Stimulation (OS) experiment was common to all subjects (see section Optimal Stimulation Experiment for details). The other experiment consisted, for all subjects, in one of three different versions of the VMA experiment (see section Visuomotor Adaptation Experiment for details). Subjects repeated each experiment two times during two different experimental sessions. During each experimental session subjects performed one repetition of each of the two experiments, always in the same order (OS first, VMA second). The different experimental sessions were held in different days, within the same week. Each experimental session lasted about 60-70 min, including setup.

\section{Experimental Setup}

All experiments were performed in the same laboratory at University College Dublin. The light in the laboratory was provided, during all experiments, artificially from overhead lamps, and lighting conditions were kept consistent during all experimental sessions of all subjects. The temperature in the laboratory was maintained at $20^{\circ} \mathrm{C}$. During all experiments, subjects sat on a chair placed in front of a computer screen placed at a distance of $1 \mathrm{~m}$ (Figure 1). The elevation of the chair was controlled so to keep the shoulder abducted at $100^{\circ}$. Subjects had the right hand strapped to a manipulandum attached to a triaxial load-cell (3A120, Interface, UK), while the wrist and the forearm were wrapped to the support plane and immobilized using self-adhesive tape. The elbow and shoulder flexion angles were fixed at $90^{\circ}$ and $60^{\circ}$, respectively. All experiments consisted in the exertion of isometric forces against the manipulandum, as instructed by a virtual scene presented on the screen. The virtual scene consisted of a gray cursor, commanded in real time by the $x$ and $y$ components of the force exerted on the manipulandum, a filled circle indicating the center of the exercise space $(0 \mathrm{~N}$ of force applied) and a target, represented by a hollow circle. The center and target circles had a radius of 0.7 or $1.2 \mathrm{~cm}$, depending on the experiment (see sections Sensory Threshold Selection and Optimal Stimulation Experiment). In all experiments targets were placed at a distance from the center equal to $7.5 \mathrm{~cm}$ on the screen, equivalent to $12 \mathrm{~N}$ force exerted in the direction of the target. Data from the load-cell were sampled at $50 \mathrm{~Hz}$. All the software constituting the virtual scene was custom developed in Labview.

\section{Sensory Threshold Selection}

At the beginning of each experimental session for each subject, a procedure for the identification of the subject- and session-specific sensory threshold (ST) was performed. Two electrodes for electrical stimulation $(5 \times 5 \mathrm{~cm}$, Valutrode Lite, Axelgaard, US) were positioned on the lateral head of the triceps brachii (TLH) muscle, that is the muscle majorly involved in reaching the upper right part of the workspace in this setup (De Marchis et al., 2018).

The electrodes were placed at about $2 / 3$ the length of the muscle belly in each direction. The ST was defined as the smallest noise-stimulation current (white Gaussian noise, bandwidth $0.1-1,000 \mathrm{~Hz}$ ) that the subject could perceive and was calculated by iteratively increasing the root mean square value (RMS) of the stimulation noise by $10 \mu \mathrm{A}$ (starting from 0 ) every $30 \mathrm{~s}$ until the subject started feeling a clear tingling sensation under the electrodes. Stimulation was administered using a voltage-driven current stimulator (Model 2200, A-M Systems, US), commanded using a custom software developed in Labview. The ST level was estimated for each subject during each experimental session.

\section{Optimal Stimulation Experiment}

The aim of the OS experiment was to determine the sessionspecific optimal stimulation level for each subject, defined as the level of sub-sensory stimulation that maximizes performance by decreasing task variability in the absence of VF. During the OS experiment subjects performed a series of reach-and-hold tasks, consisting of reaching for a target of $0.7 \mathrm{~cm}$ of diameter positioned in the upper right side of the screen (where the TLH is active) and then holding the cursor as close as possible to the center of the target for $30 \mathrm{~s}$ (Figure 1). The VF was projected on the screen only during the reaching phase and during the first $10 \mathrm{~s}$ of the hold phase and was then removed for the remaining $20 \mathrm{~s}$ of the hold phase.

During each task, subjects received sub-sensory noise stimulation on their TLH muscle at six different current levels, equal to $0 \%$ (no current), $50,60,70,80$, or $90 \%$ of their ST. Subjects experienced each level of sub-sensory stimulation five times in a random order, for a total of 30 repetitions (6 current levels $\times 5$ times). The session-specific OS level was estimated at the end of each OS experiment as the percentage of ST (excluding 0\% ST) yielding the smallest average (across the 5 repetitions for each percentage) standard deviation in the Cartesian distance between the 


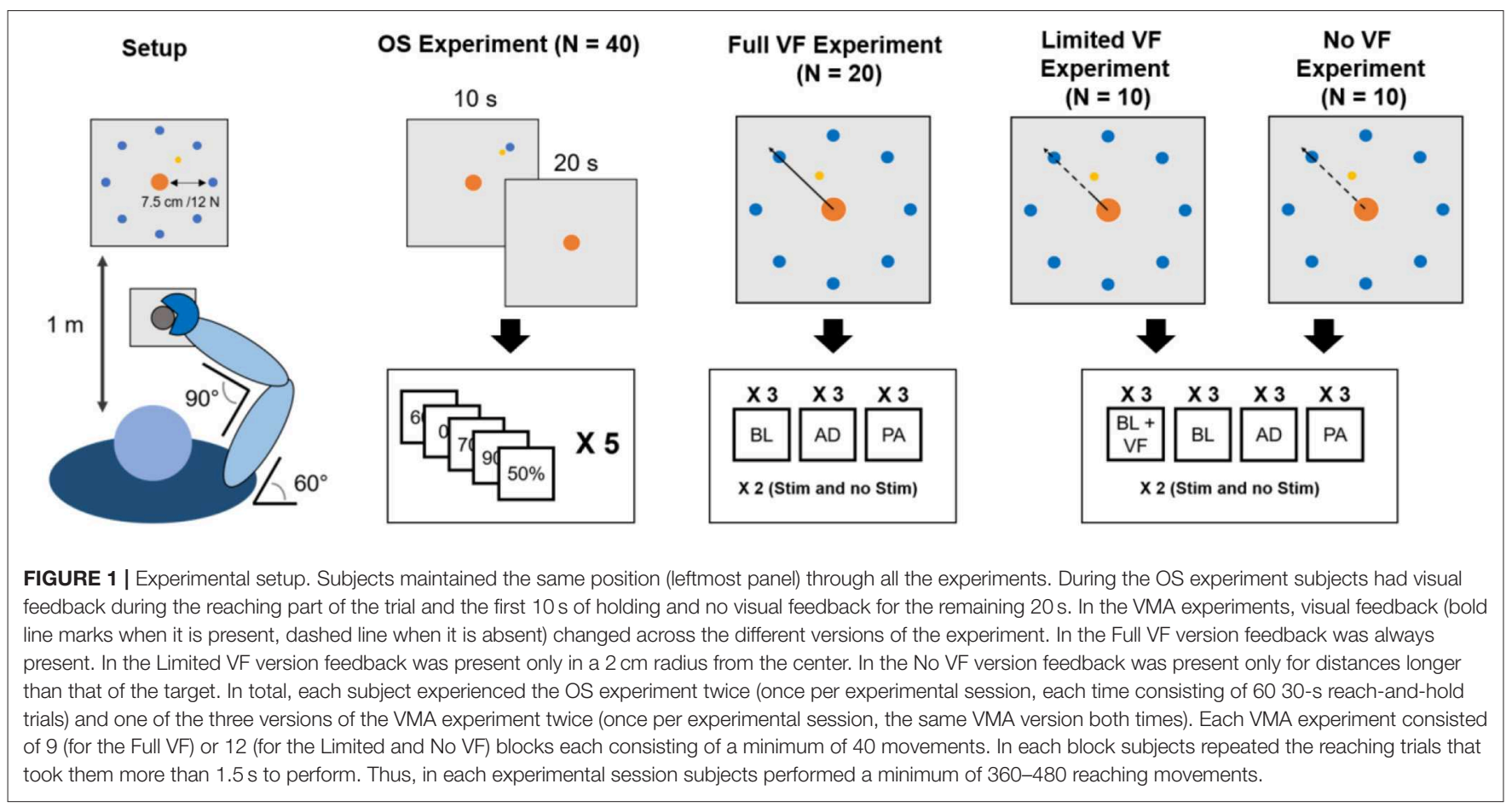

cursor and the target during the $20 \mathrm{~s}$ of the hold phase of the task where the visual feedback was not present (stdDist). Additional analyses were performed in post processing. Specifically, we checked for statistically significant differences (Wilcoxon's signed rank test, $\alpha=0.05$ ) in the average stdDist between OS and 0\% ST across all subjects. We then analyzed the distribution of the OS percentages across the different stimulation levels, for both OS experiments of all subjects. Finally, we analyzed the relationship between the stimulating current and the motor performance by fitting a first order polynomial, using a least square algorithm, on the average stdDist values relative to each stimulation intensity. The quality and significance of the fitting was evaluated by calculating Pearson's coefficient $\rho$.

\section{Visuomotor Adaptation Experiment}

All three versions of the VMA experiment consisted of isometric reaching movements where the subjects were asked to drive the cursor toward a random target (diameter $1.2 \mathrm{~cm}$ ) presented at $7.5 \mathrm{~cm}(12 \mathrm{~N})$ from the center. Targets were presented in 8 different directions covering the whole $360^{\circ}$ of the workspace at angular intervals of $45^{\circ}$ (Figure 1). Subjects performed their assigned version of the VMA experiment immediately after the OS one, in both experimental sessions. The versions of the VMA experiment differed only in the VF that was provided to the subject during the reaching tasks. Twenty subjects ( 9 females) performed the VMA experiment with continuous view of the movements of the cursor they were driving (Full VF). Ten subjects ( 2 females) performed the VMA experiment while receiving $\mathrm{VF}$ of the movement of the target only up to $2 \mathrm{~cm}$ $(3.3 \mathrm{~N})$ from the center of the virtual scene (Limited $V F)$. Finally,
10 subjects ( 8 females) performed the experiment with no VF (No $V F$ ) on the movement of the cursor during the trajectory. In the No VF experiment subjects were shown the cursor only between 0 and $0.5 \mathrm{~cm}(0.7 \mathrm{~N})$ from the center and after exceeding the distance to the center of the target $(7.5 \mathrm{~cm}, 12 \mathrm{~N})$. Thus, in the No VF experiment subjects were able to see the cursor only when its position exceeded the distance between the center of the workspace and the center of the target and therefore received feedback only on the result of their reaching trial. The VMA experiment consisted of 9 blocks during which the VF condition was applied. In the first 3 blocks (baseline, BL1-BL3) participants were asked to reach to the 8 targets positioned in a compasslike configuration for 5 times in a random order (Figure 1). During these and subsequent blocks, subjects were instructed to reach for the targets at a self-selected speed but in a time smaller than $1.5 \mathrm{~s}$ and they were given positive feedback (consisting in the target becoming green) if they were able to reach for the target in $<1.5 \mathrm{~s}$, and negative feedback (consisting in the target becoming red) otherwise. In all the trials the movement time was not restricted, and subjects were presented a new target only when the current target had been reached. As a result, subjects were forced to explore the space until they were able to reach the current target before being shown the following one. The feedback on the duration of each trial indicated by the change in color of the target was present in all three VF conditions. The targets for which a subject received negative feedback were appended and repeated at the end of the block, thus making each block consisting of 40 movements plus the repetition of all the negative-feedback movements. After the BL blocks, subjects performed three adaptation blocks (AD1-AD3) where they were asked to reach for the targets while adapting to a $45^{\circ}$ clockwise 


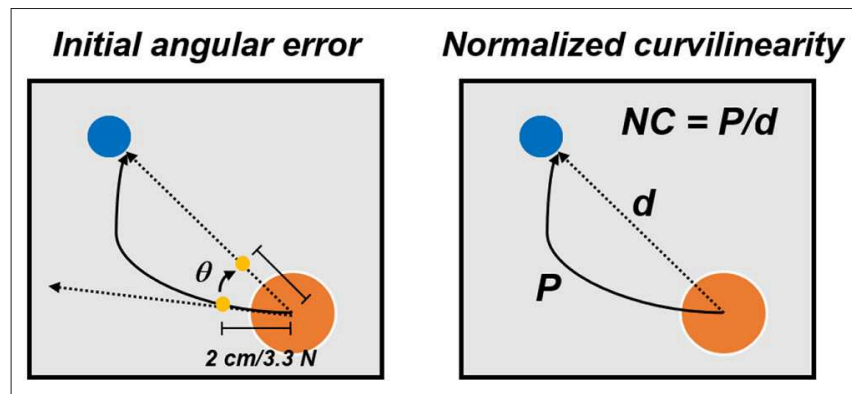

FIGURE 2 | Performance metrics for the VMA experiment. The initial angular error (left) was calculated, for each movement repetition, as the angle between the actual and optima trajectories at $2 \mathrm{~cm}$ from the center of the workspace. The normalized curvilinearity (right) was calculated, for each movement repetition, as the ratio between the actual movement path and the ideal one.

rotation applied to the mapping between the force sensor and the virtual scene. As for the BL blocks, the only instruction that the subjects were given was to try to obtain positive feedbacks on their movements by reaching for the targets in $<1.5 \mathrm{~s}$. Also in this case, subjects performed 5 repetitions of all 8 targets in a randomized order (40 tasks), and repeated the targets for which they received negative feedback at the end of the trial. Thus, after the repeated trials, each subject would have effectively adapted to 40 movements in each adaptation block.

Finally, subjects performed three unperturbed postadaptation blocks (PA1-PA3) that were used to washout the adapted motor plan. These blocks were identical to the $\mathrm{BL}$ ones. Subjects who performed the Limited VF and No VF VMA versions also experienced 3 additional blocks before the BL ones, that consisted of unperturbed baseline blocks with full VF (BLVF). The aim of these blocks was to allow the subjects to practice and fully understand the task before the limitation to the VF was applied. Subjects performed their assigned VMA experiment in both experimental sessions, once while receiving sub-sensory stimulation (through all the 9 blocks of the experiment) at the OS level calculated in that same experimental session (Stim), and once without stimulation (NoStim), in a random order. Participants were blinded to the condition. For all three versions of the VMA experiment, half of the assigned subjects performed the Stim condition in the first experiment and the other half in the second experiment. For each reaching repetition, we analyzed the center-out portion of the movement, from the moment in which the cursor exited the origin target to the moment it reached the goal target. Each center-out movement was extracted and length-normalized over 100 data points. We analyzed the trajectory data by means of two metrics (Figure 2): the initial angular error (IAE) and the normalized curvilinearity (NC). The IAE was calculated as the angle between the straight line connecting the ideal path and the actual path of the movement at $2 \mathrm{~cm}$ from the origin. This distance was selected because subjects performing the Limited VF experiment had the VF removed after $2 \mathrm{~cm}$, thus, for them, this metric represents the angular error before losing VF. The NC was defined as the ratio between the actual distance covered by the cursor between the center and the target and the length of the straight line connecting the center and the target. The IAE is intended to capture the error in movement planning before the onset of potential compensations, while the NC metric accounts for both the initial movement error and the changes in motor plan that the subject undergoes to compensate for the shooting error. The analysis of IAE and NC was performed on the first 40 movements of each block (thus excluding the repeated trials in each blocks). Moreover, the analysis were differentially performed on all targets together and by considering only the targets were the triceps are active (that are, using a compass notation, targets N, NE, and E, as estimated in De Marchis et al., 2018, using the same experimental setup) or the targets were the triceps are not involved (all targets excluding $\mathrm{N}, \mathrm{NE}$, and E). For all these three targets-group analyses (all targets, triceps-active targets, and remaining targets) a statistical analysis was used to compare the values of NC and IAE between the two stimulation conditions. This analysis was based on Wilcoxon's signed rank test with significance level $\alpha=0.05$ and Bonferroni-Holm's correction for multiple comparisons over the different blocks.

As an additional analysis on the effect of the stimulation on task performance we compared, for all three versions of the VMA experiment, the number of repeated trials (thus the number of errors) across subjects in the first block of adaptation (AD1, where negative-feedback trials were more expected due to the introduction of the perturbation) between the Stim and NoStim conditions. This comparison was based on a Wilcoxon's signed rank test with significance level $\alpha=0.05$.

Finally, we analyzed differences in movement time across the different VF and stimulation conditions for the BL3 and AD1 blocks. The analysis on BL3 was carried out mainly to assess baseline differences in movement time across the three VF conditions, while the analysis on $\mathrm{AD} 1$ was performed mainly to assess the effect of the stimulation on movement time during the early stages of adaptation. The analysis was performed, for both blocks, on the first 40 movements, without accounting for the repeated ones. We tested for statistically significant differences in movement time across VF conditions (regardless of the stimulation condition) using ANOVA. On the other hand, given that our hypothesis is that the stimulation will have different effects depending on the VF condition, we tested for statistically significant differences in movement time across stimulation conditions separately for each VF condition using Wilcoxon's signed rank test with significance level $\alpha=0.05$.

\section{RESULTS}

\section{OS Experiment}

The results of the OS experiments performed by the subjects in the two experimental sessions were pooled together in the analysis. Thus, the 80 instances $(40$ subjects $\times 2$ experimental sessions) were treated as independent measures. As expected from similar experiments (Magalhaes and Kohn, 2012, 2014; Severini and Delahunt, 2018), we consistently observed a decrease in accuracy during the hold-phase of the OS task when the VF was removed (example for one trial of one subject in Figure 3A). From the analysis of the OS levels, considering also the trials where no current was applied $(0 \%)$, we observed that 

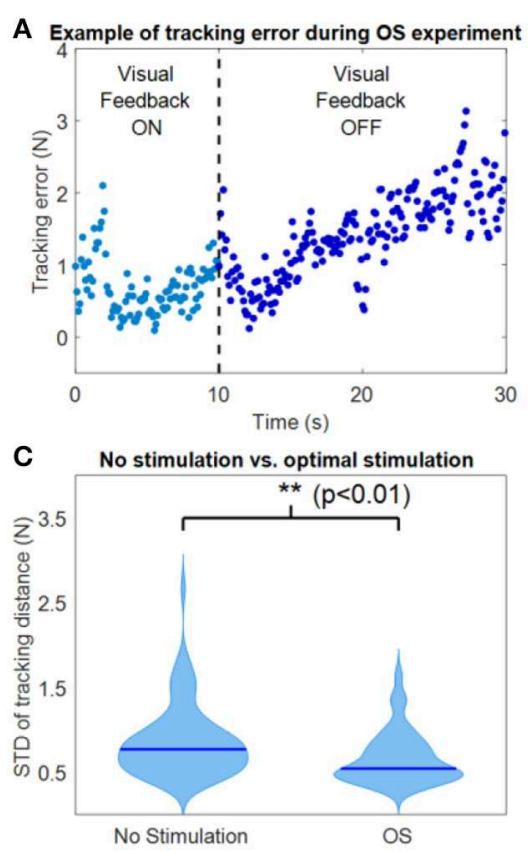

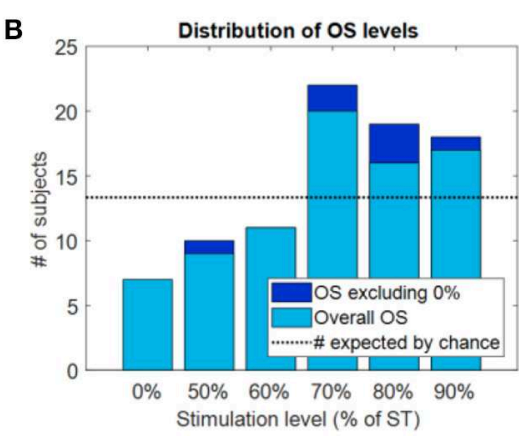

D

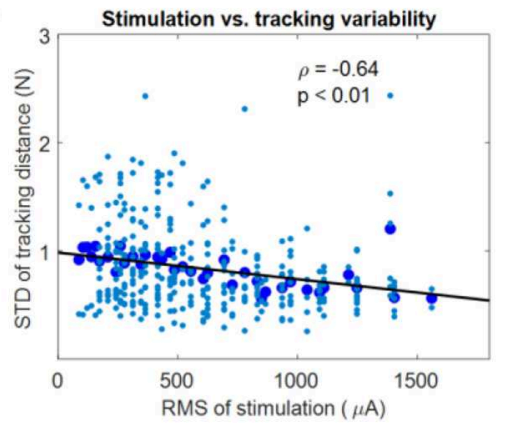

FIGURE 3 | Results of the OS experiment. (A) Example of tracking error during a representative instance of the OS experiment. Movement variability around the target position increased as visual feedback was removed. (B) Distribution of the OS values, both including (light blue) and excluding (dark blue) the $0 \%$ level. (C) Violin plots of the tracking variability between OS values and $0 \%$ (no stimulation) values. ${ }^{\star \star}$ Indicates significant differences (Wilcoxon's signed rank test) with $p<0.01$.

(D) Correlation between the RMS of stimulation and the STD of the tracking distance during the OS experiment.

in 7 instances out of 80 , the average stdDist was lower for $0 \%$ stimulation than for a stimulation level above $0 \%$. This accounts for $8.75 \%$ of the instances, against a value expected by chance of $13.33 \%$ (Figure 3B). For the instances in which the $0 \%$ level presented the lowest average value of stdDist across the task repetitions, the value of OS was selected as the value of actual stimulation (thus above 0\%) which yielded the lowest average stdDist (Figure 3B). The OS levels were mostly distributed toward percentages close to the ST (Figure 3B) with 59 out of 80 OS levels observed for percentages of ST above $70 \%$. We observed statistically significant lower values of stdDist for OS with respect to $0 \%$ stimulation ( $p<0.01$ using Wilcoxon's signed rank test), also considering the instances were $0 \%$ yielded the average lower stdDist results (Figure 3C). Finally, we analyzed the correlation between the RMS of the stimulation current and the stdDist metric.

We observed a negative correlation (Figure 3D) between the average stdDist metric (averaged across all repetitions associated with a specific current level across subjects) and the relative RMS values of stimulation current, characterized by a significant $(p<0.001)$ linear fitting with $\rho=-0.64$. This fitting indicates that, in our dataset, the performance increases with the RMS of the stimulation.

\section{VMA Experiments}

The results for the Full VF version of the VMA experiment (Figure 4) were in line with what had been observed in literature (Krakauer et al., 1999) (Figure 4A). Subjects presented marked movement errors, reflected in both the IAE and NC metrics, in the first block of perturbation (AD1) that were compensated over time. After-effects opposite to the direction of the original perturbation (in the IED) were present at the beginning of the post-adaptation phase and quickly vanished by the end of the experiment. When comparing the Stim and NoStim conditions, we were not able to observe substantial differences in trends in both metrics, such as different values of IED or $\mathrm{NC}$ at the beginning of AD1 or at the end of AD3. These differences would have indicated a higher/lower initial error and a higher/lower level of compensation of the error, respectively. Instead, both conditions presented remarkably similar trends in both metrics when considering all targets (Figure 4B), only the targets where the triceps are active (Figure 4C), and the targets where the triceps were not active (Figure 4D). We did not observe statistically significant differences between the two conditions in any of the blocks of the different targets-group analyses. Similarly, we did not observe significant differences in the number of errors made by the subjects in AD1 between the two stimulation conditions for all the target groupings (rightmost panel, Figures 4B-D).

In the Limited VF version of the VMA experiment (Figure 5), trajectories were characterized by initial shooting errors followed by abrupt deviations once the VF was removed (Figure 5A). As the $\mathrm{AD}$ blocks progressed, subject showed decreased shooting errors (also captured by a progressive decrease in IAE and NC) but still exhibited abrupt modifications in their trajectories once the feedback was removed. When comparing 


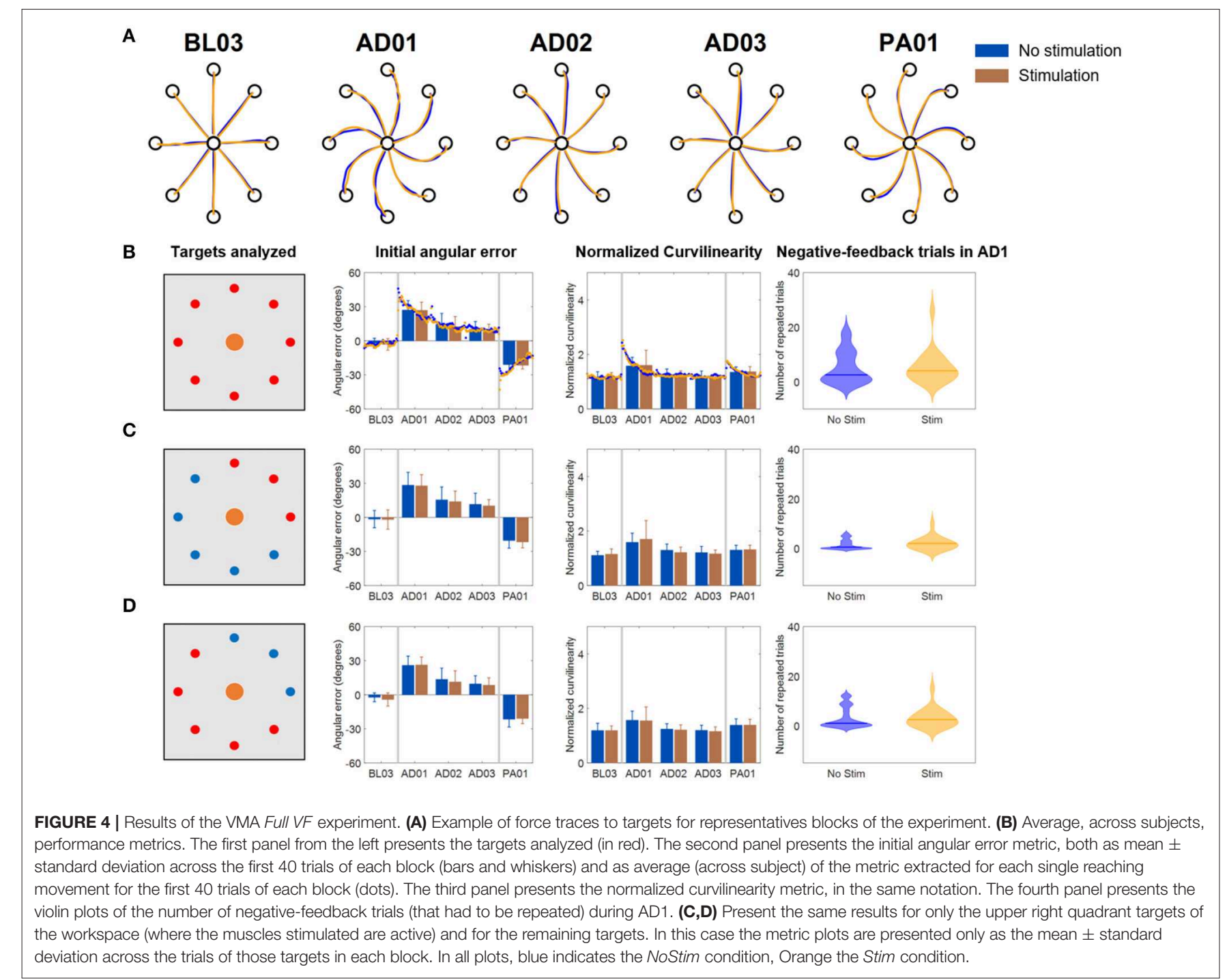

the Stim and NoStim conditions we observed a qualitative trend where Stim trials were characterized by higher initial values of IAE and NC at AD1 with respect to NoStim. The two conditions exhibited similar values on both metrics at AD3. The trends observed appeared to be present on all targets, regardless of groupings (Figures 5B-D). Nevertheless, we did not observe statistically significant differences between the two stimulation conditions in any of the blocks of the different targets-group analyses. Finally, we did not observe significant difference in the number of errors at $\mathrm{AD} 1$ between the two conditions.

In the No VF version of the VMA experiment (Figure 6), once again we observed initial changes in both metrics at AD1 due to the rotation. These changes were compensated over the trials even without VF (consistently with what shown in Scheidt et al., 2005) although to a smaller level with respect to the Full VF experiment (Figures 4A, 6A). Also in this case, the adaptation behaviors were reflected in both metrics. Subjects, in fact, were able to decrease their IAE and NC values over the various repetitions of the No VF task even without feedback on their actual trajectory.

We did not observe differences in the behavior of the IAE and NC metrics between the two stimulation conditions, either for all the targets or for the different groupings. This was reflected also in the absence of statistically significant differences between the two stimulation conditions in all the blocks for the different targets-group analyses.

However, the NoStim condition presented a significant higher number of reaching errors at AD1 with respect to the Stim condition that was observed for all the targets togethers $(p=0.046$, Figure 6B) and for the grouping representing only the targets were the triceps were active $(p=0.043$, Figure 6C).

The analysis of the movement time (Figure 7) at BL3 unraveled a difference in the strategy that the participants employed for reaching to the targets between the VF conditions. In fact, although in all VF conditions subjects were instructed to reach for the target at a comfortable speed while taking 
A

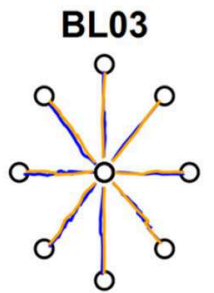

B

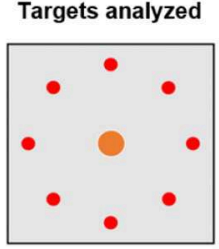

C

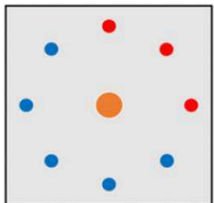

D

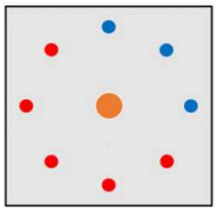

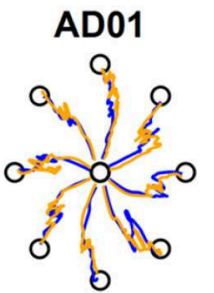

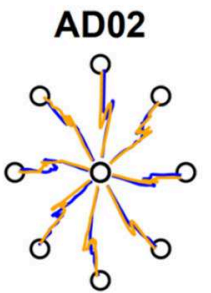

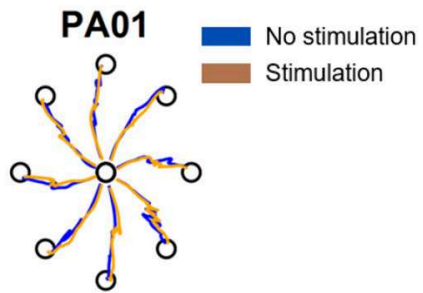

Normalized Curvilinearity Negative-feedback trials in AD1
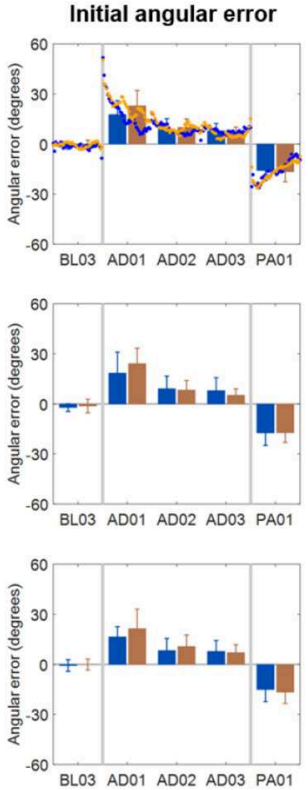
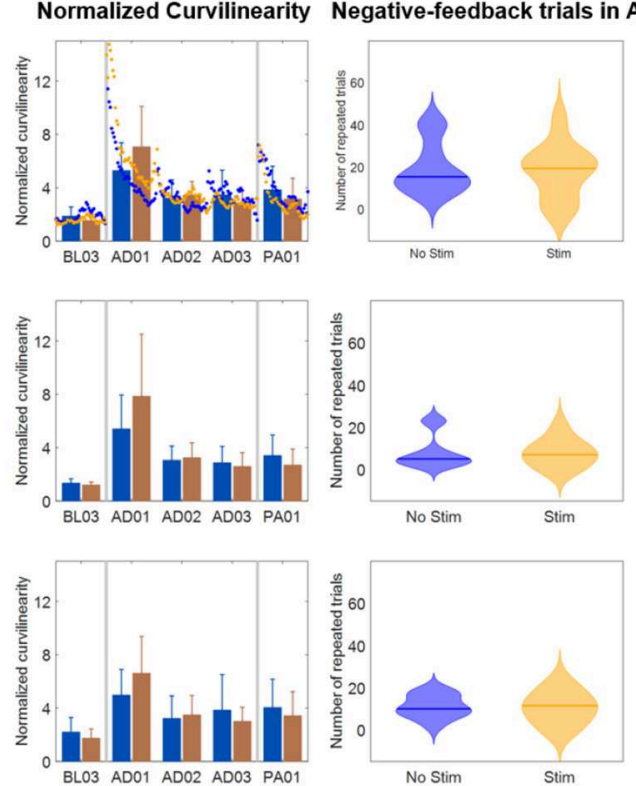

FIGURE 5 | Results of the VMA Limited VF experiment. (A) Example of force traces to targets for representatives blocks of the experiment. (B) Average, across subjects, performance metrics. The first panel from the left presents the targets analyzed (in red). The second panel presents the initial angular error metric, both as mean \pm standard deviation across the first 40 trials of each block (bars and whiskers) and as average (across subject) of the metric extracted for each single reaching movement for the first 40 trials of each block (dots). The third panel presents the normalized curvilinearity metric, in the same notation. The fourth panel presents the violin plots of the number of negative-feedback trials (that had to be repeated) during AD1. (C,D) Present the same results for only the upper right quadrant targets of the workspace (where the muscles stimulated are active) and for the remaining targets. In this case the metric plots are presented only as the mean \pm standard deviation across the trials of those targets in each block. In all plots, blue indicates the NoStim condition, Orange the Stim condition.

$<1.5 \mathrm{~s}$, subjects undergoing the Full $V F$ condition took longer to reach for the target with respect to the Limited $V F$ and No VF conditions, and this difference was found to be statistically significant ( $p<0.001$ based on ANOVA analysis). We did not find statistically significant differences between the two stimulation conditions at BL3 for any of the three VF conditions, based on individual Wilcoxon's signed rank tests. At AD1, all three groups increased their movement time while undergoing adaptation, in a way that was consistent across VF conditions, as reflected by the fact that we did not observe statistically significant differences for VF $(p=0.45)$. When comparing for the stimulation conditions, we only found statistically significant differences $(p=0.049)$ for the No VF condition, where subjects undergoing stimulation were able to reach for the targets in less time $(1.28 \mathrm{~s}$, median across subjects) with respect to their same performance while not stimulated ( $1.65 \mathrm{~s}$, median across subjects). This latter result is in accordance with what observed in the analysis of the negative feedback trials.

\section{DISCUSSION}

In our results we observed that sub-sensory electrical stimulation was associated with smaller movement variability during the phase of the OS experiment where VF was not available and task performance depended solely on proprioceptive feedback. Moreover, we observed a correlation between stimulation current and movement variability whereas higher current levels were associated with better task performance across subjects. These results, taken together, further confirm that sub-sensory electrical stimulation can improve task performance in tasks were proprioception is the primary feedback modality (Gravelle et al., 2002; Ross and Guskiewicz, 2006; Collins et al., 2014; Severini and Delahunt, 2018).

On the other hand, we observed only small evidence of an effect of the stimulation during the VMA experiments, that was mainly characterized by a significant decrease in negativefeedback movements (that are movements that took more than $1.5 \mathrm{~s}$ for the subject to complete) and movement time 
A BL03

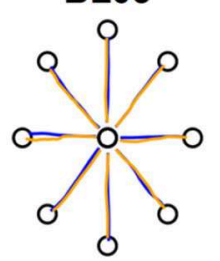

B

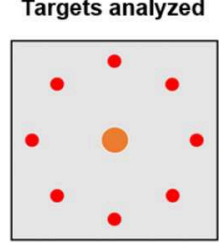

C

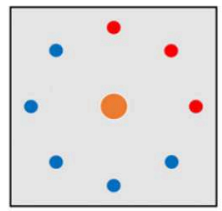

D

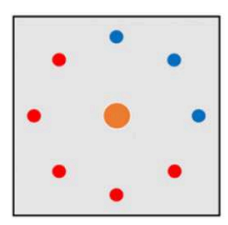

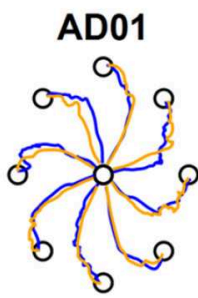

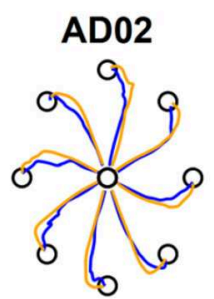

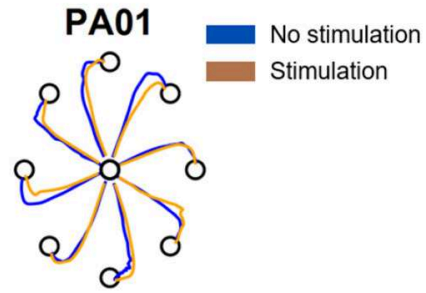

Initial angular error
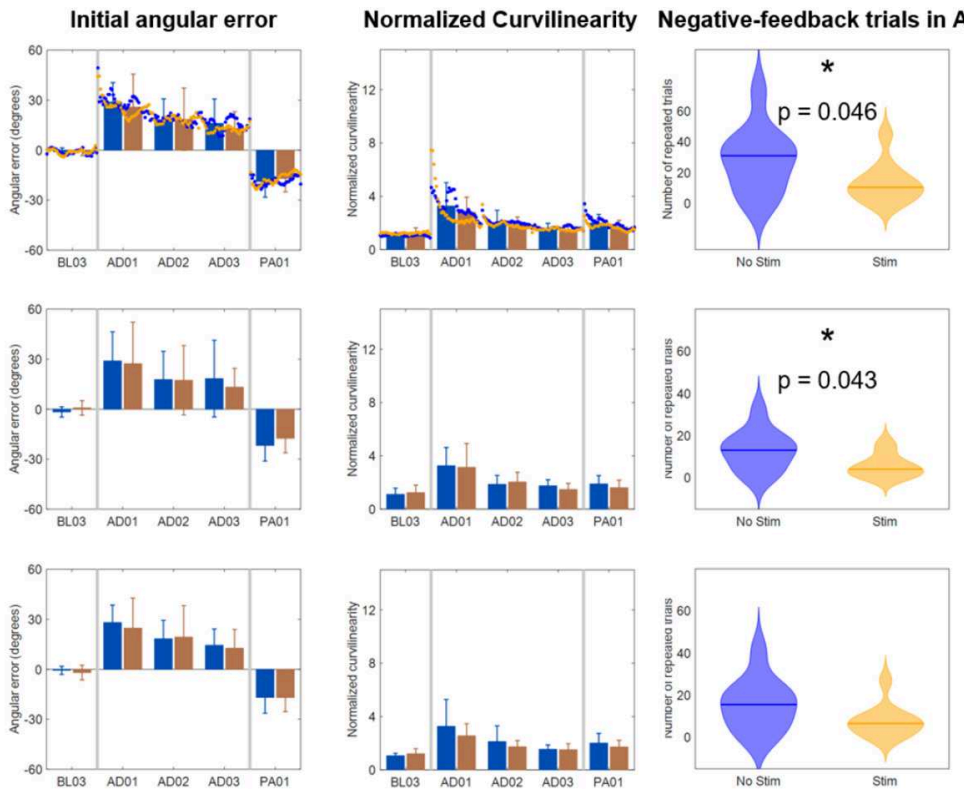

FIGURE 6 | Results of the VMA No VF experiment. (A) Example of force traces to targets for representatives blocks of the experiment. (B) Average, across subjects, performance metrics. The first panel from the left presents the targets analyzed (in red). The second panel presents the initial angular error metric, both as mean \pm standard deviation across the first 40 trials of each block (bars and whiskers) and as average (across subject) of the metric extracted for each single reaching movement for the first 40 trials of each block (dots). The third panel presents the normalized curvilinearity metric, in the same notation. The fourth panel presents the violin plots of the number of negative-feedback trials (that had to be repeated) during AD1. *Indicates significant differences (Wilcoxon's signed rank test) in the number of negative-feedback trials with $p<0.05[p=0.046$ in (B) and $p=0.043$ in (C)]. (C,D) Present the same results for only the upper right quadrant targets of the workspace (where the muscles stimulated are active) and for the remaining targets. In this case the metric plots are presented only as the mean \pm standard deviation across the trials of those targets in each block. In all plots, blue indicates the NoStim condition, Orange the Stim condition.

between the two stimulation conditions during the first block of adaptation for the subjects that performed the No VF version of the experiment. When the VF of the trajectory was present, completely or partially, we did not observe substantial differences in task performance, as captured by two different metrics, between the Stim and NoStim conditions other than a qualitative (not statistically significant) slight decrease in task performance during AD1 for the Limited VF group. In the following, we will further discuss upon these results.

The results of the OS experiment provide, in this study, the strongest evidence of the effectiveness of sub-sensory stimulation in boosting proprioception and influence task performance. In the OS experiment we did not observe a clear SR behavior, characterized by a U-shaped relationship between the change in performance and the intensity of the stimulation (Collins et al., 1995). Such behavior is unlikely to appear in a group analysis (Bates, 1996; Severini and Delahunt, 2018), given the differences in ST across subjects and across different sessions for the same subjects that have been observed in this and previous studies (Magalhaes and Kohn, 2012, 2014). Nevertheless, we did observe a significant negative correlation between the stimulation intensity and the tracking error (Figure 3D), suggesting that subsensory stimulation is more effective as its intensity increases. This linear relationship does not rule out the presence of a SR-like behavior, but hints that such behavior may arise by considering stimulation intensities that are above the ST of subjects. On the other hand, stimulating currents above ST could lead to additional confounding factors affecting motor task performance related to the increase in attention or arousal, and the few studies that investigated the use of supra-sensory stimulation levels in humans found that it leads to an overall decrease in performance (Iliopoulos et al., 2014; Severini and Delahunt, 2018). The results of the OS experiment support the design choice of using subsensory stimulation levels close but below ST (frequently $90 \%$ of ST) that is often employed in similar studies (Gravelle et al., 2002; Magalhaes and Kohn, 2012, 2014). 

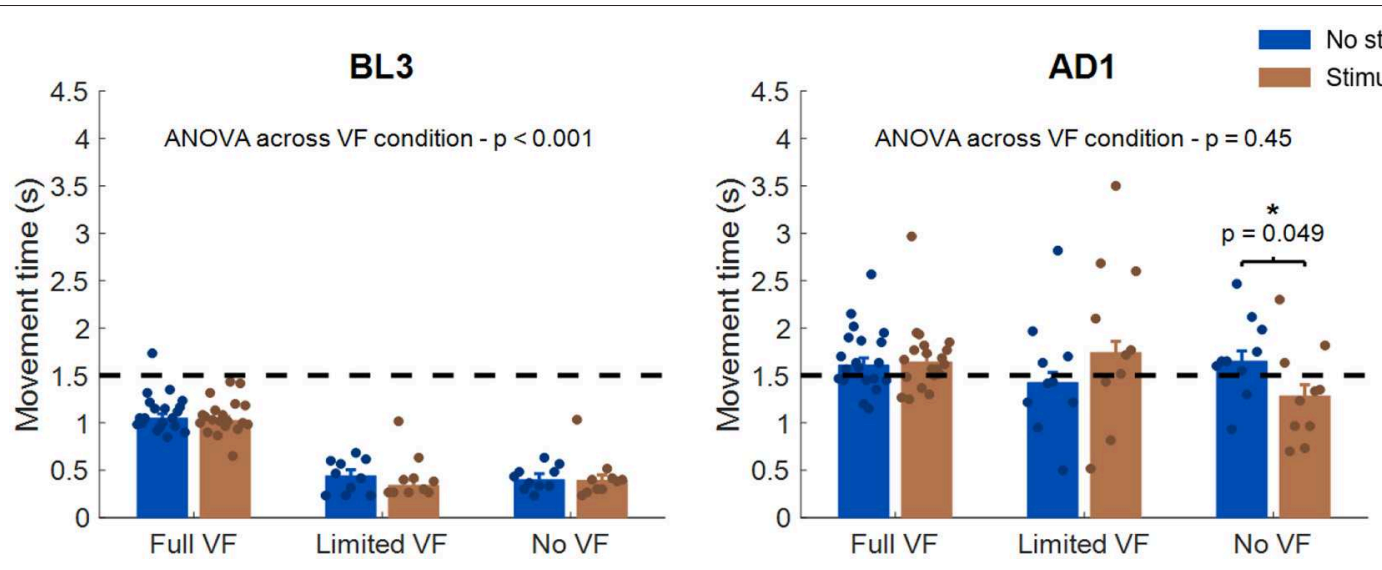

FIGURE 7 | Analysis of movement time across VF and stimulation conditions. The left panel presents the results for BL3, the right panel for AD1. In each panel, each bar represents the median and standard error of the median movement time for each stimulation and VF condition. The dots represent the median values for each individual subject. The statistical analysis across VF conditions was based on ANOVA, while the statistical analysis between stimulation conditions was performed independently for each VF condition using Wilcoxon's signed rank test. ${ }^{*} p<0.05$. The dotted black line in each panel represents the $1.5 \mathrm{~s}$ threshold that was set to mark negative-feedback trials.

In contrast with the results obtained in the OS experiment, we observed little evidence of an effect of the stimulation during the different VMA experiments. In the Full VF version of the experiment, the adaptation patterns were remarkably similar between the two stimulation conditions. We observed some small differences in performance between the two stimulation conditions in the first block of adaptation for both the Limited $V F$ and the No VF versions of the experiment. In the Limited $V F$ experiment we qualitatively observed higher values in both performance metrics during AD1 for the Stim condition, nevertheless these differences were not statistically significant. In the No $V F$ experiment we did not observe differences in trends between the two metrics, but the Stim condition was characterized by a statistically significant smaller number of negative-feedback trials and a statistically significant smaller movement time, especially for the targets of the upper right quadrant, where the muscle undergoing stimulation was active. Both the trends that we observed in the Limited VF and No VF experiments could be potentially explained by the stimulation impacting the weighting process between proprioceptive and visual feedbacks that happens during reaching tasks in general, and motor adaptations in particular. Previous studies have shown that different feedback modalities mix flexibly during the execution of voluntary movements and during motor adaptations (Sober and Sabes, 2003, 2005; Scheidt et al., 2005; Shabbott and Sainburg, 2010). While visual feedback is responsible for estimating the limb position required in the planning of the movement trajectory, proprioception contributes in generating the necessary feedforward commands required for movement execution (van Beers et al., 2002; Sober and Sabes, 2003, 2005). Primary and secondary muscle spindles have been observed to increase their firing rates during isometric contractions (Edin and Vallbo, 1990), indicating that these afferents encode information on muscular state even if the muscles are not changing in length. A previous study on spindles behavior during visuomotor adaptations has shown that adaptation leads to a progressive decrease in the activity of the spindles (Jones et al., 2001). The authors linked this result to the fact that adaptation to visuomotor rotations is achieved by updating the internal models mapping the kinematics of the movement, a process relying mostly on visual and less on proprioceptive feedback (Krakauer et al., 1999; Krakauer, 2009), as confirmed also in a study involving individuals with proprioceptive deficits (Lajoie et al., 1992). Decreasing the weight of the spindles' information during visuomotor adaptation would then help resolving the conflict between the visual and proprioceptive maps that the perturbation induces (Jones et al., 2001). This re-weighting of proprioceptive information has been shown to happen centrally, at the level of the somatosensory cortex, rather than at the spinal level (Bernier et al., 2009), and to be more prominent at the beginning of the adaptation period and then alleviated as the adaptation converges.

Thus, a potential interpretation of our results could be that as the activity of the spindles is down-regulated at the beginning of adaptation, the supposed enhancement of such activity by the stimulation would effectively clash with the sensory re-weighting process. This clash, in the Limited VF experiment, where VF of the shooting error is provided but proprioceptive feedback is still necessary for successfully completing the task, could translate in bigger initial errors as the stimulation supposedly antagonizes the spindle down-regulation. The fact that a similar effect is not present if the Full VF experiment could be explained by the primacy of VF over proprioception during visuomotor adaptations that bypasses the potential effects of the stimulation. On the other hand, in the No VF experiment, where proprioception is the only available feedback modality, the supposed proprioceptive boost obtained through the stimulation may lead to increased feedback reliability that may translate in a smaller movement time and smaller number of negativefeedback trials. In fact, since the movement time is not restricted 
and the new target is only shown after reaching the available target, subjects, after the initial shooting error, must explore the movement space relying only on their proprioceptive feedback in order to advance in the trial. These explanations, although plausible, cannot be fully confirmed from our results due to: (i) the fact that we do not directly measure sensory re-weighting in our experiments; (ii) the small sample examined, that is the main limitation of the study herein presented. Another limitation of this study, that could also help explain the differences in stimulation effectiveness that we observed between the OS and VMA experiments, is represented by the fact that we selected the optimal stimulation level based on the performance during the holding phase of the OS experiment and then tested it during a reaching task in the VMA experiments. In a literature review recently published by Shadmehr (2017) the author observed that these two tasks (holding and reaching), similarly to what happens during ocular movements, employ different neural circuitries. In this interpretation, the discrepancy in stimulation effectiveness that we observe could be explained by an experimental design flaw where we used optimal currents derived from the holding task in a task that employs different neural circuits. Nevertheless, although there is evidence on the different nature of neural inputs during reaching and holding, no information is available on if and how proprioceptive feedback is processed differently between these two tasks.

To summarize, the results presented in this study further support the hypothesis that sub-sensory currents applied to the

\section{REFERENCES}

Bates, B. T. (1996). Single-subject methodology: an alternative approach. Med. Sci. Sports Exerc. 28, 631-638. doi: 10.1249/00005768-199605000-00016

Bernier, P. M., Burle, B., Vidal, F., Hasbroucq, T., and Blouin, J. (2009). Direct evidence for cortical suppression of somatosensory afferents during visuomotor adaptation. Cereb. Cortex 19, 2106-2113. doi: 10.1093/cercor/bhn233

Collins, A., Blackburn, T., Olcott, C., Jordan, J. M., Yu, B., and Weinhold, P. (2014). A kinetic and kinematic analysis of the effect of stochastic resonance electrical stimulation and knee sleeve during gait in osteoarthritis of the knee. J. Appl. Biomech. 30, 104-112. doi: 10.1123/jab.2012-0257

Collins, J. J., Chow, C. C., and Imhoff, T. T. (1995). Aperiodic stochastic resonance in excitable systems. Phys. Rev. E Stat. Phys. Plasmas Fluids Relat. Interdiscip. Topics 52, R3321-R3324. doi: 10.1103/PhysRevE.52.R3321

Collins, J. J., Priplata, A. A., Gravelle, D. C., Niemi, J., Harry, J., and Lipsitz, L. A. (2003). Noise-enhanced human sensorimotor function. IEEE Eng. Med. Biol. Mag. 22, 76-83. doi: 10.1109/MEMB.2003.1195700

Cordo, P., Inglis, J. T., Verschueren, S., Collins, J. J., Merfeld, D. M., Rosenblum, S., et al. (1996). Noise in human muscle spindles. Nature 383, 769-770. doi: $10.1038 / 383769 \mathrm{a} 0$

De Marchis, C., Di Somma, J., Zych, M., Conforto, S., and Severini, G. (2018). Consistent visuomotor adaptations and generalizations can be achieved through different rotations of robust motor modules. Sci. Rep. 8:12657. doi: 10.1038/s41598-018-31174-2

Edin, B. B., and Vallbo, A. B. (1990). Muscle afferent responses to isometric contractions and relaxations in humans. J. Neurophysiol. 63, 1307-1313. doi: 10.1152/jn.1990.63.6.1307

Fleishman, E. A., and Rich, S. (1963). Role of kinesthetic and spatialvisual abilities in perceptual-motor learning. J. Exp. Psychol. 66, 6-11. doi: 10.1037/h0046677

Gammaitoni, L. (1995). Stochastic resonance and the dithering effect in threshold physical systems. Phys. Rev. E Stat. Phys. Plasmas Fluids Relat. Interdiscip. Topics 52, 4691-4698. doi: 10.1103/PhysRevE.52.4691 surface of the muscles affect proprioceptive feedback during movement, but this effect appears to be limitedly beneficial for task performance only in tasks where proprioception is the primary feedback modality.

\section{DATA AVAILABILITY STATEMENT}

The datasets generated for this study are available on request to the corresponding author.

\section{ETHICS STATEMENT}

The studies involving human participants were reviewed and approved by Ethic Committee, University College Dublin. The patients/participants provided their written informed consent to participate in this study.

\section{AUTHOR CONTRIBUTIONS}

AC and GS conceived the study, interpreted the results, and drafted the manuscript. $\mathrm{CG}, \mathrm{MH}$, and GS designed the experiments and analyzed the data. $\mathrm{CG}$ and $\mathrm{MH}$ performed the data collections.

\section{FUNDING}

This study was partially funded by the UCD Seed Fund \#SF1303.
Gravelle, D. C., Laughton, C. A., Dhruv, N. T., Katdare, K. D., Niemi, J. B., Lipsitz, L. A., et al. (2002). Noise-enhanced balance control in older adults. Neuroreport 13, 1853-1856. doi: 10.1097/00001756-200210280-00004

Iliopoulos, F., Nierhaus, T., and Villringer, A. (2014). Electrical noise modulates perception of electrical pulses in humans: sensation enhancement via stochastic resonance. J. Neurophysiol. 111, 1238-1248. doi: 10.1152/jn.00392.2013

Jones, K. E., Wessberg, J., and Vallbo, A. (2001). Proprioceptive feedback is reduced during adaptation to a visuomotor transformation: preliminary findings. Neuroreport 12, 4029-4033. doi: 10.1097/00001756-200112210-00035

Krakauer, J. W. (2009). Motor learning and consolidation: the case of visuomotor rotation. Adv. Exp. Med. Biol. 629, 405-421. doi: $10.1007 / 978-0-387-77064-2 \_21$

Krakauer, J. W., Ghilardi, M. F., and Ghez, C. (1999). Independent learning of internal models for kinematic and dynamic control of reaching. Nat. Neurosci. 2, 1026-1031. doi: 10.1038/14826

Lajoie, Y., Paillard, J., Teasdale, N., Bard, C., Fleury, M., Forget, R., et al. (1992). Mirror drawing in a deafferented patient and normal subjects: visuoproprioceptive conflict. Neurology 42, 1104-1106. doi: 10.1212/WNL.42.5.1104

Magalhaes, F. H., and Kohn, A. F. (2012). Imperceptible electrical noise attenuates isometric plantar flexion force fluctuations with correlated reductions in postural sway. Exp. Brain Res. 217, 175-186. doi: 10.1007/s00221-011-2983-6

Magalhaes, F. H., and Kohn, A. F. (2014). Effectiveness of electrical noise in reducing postural sway: a comparison between imperceptible stimulation applied to the anterior and to the posterior leg muscles. Eur. J. Appl. Physiol. 114, 1129-1141. doi: 10.1007/s00421-014-2846-5

Mendez-Balbuena, I., Huidobro, N., Silva, M., Flores, A., Trenado, C., Quintanar, L., et al. (2015). Effect of mechanical tactile noise on amplitude of visual evoked potentials: multisensory stochastic resonance. J. Neurophysiol. 114, 2132-2143. doi: $10.1152 /$ jn.00457.2015

Mendez-Balbuena, I., Manjarrez, E., Schulte-Monting, J., Huethe, F., Tapia, J. A., Hepp-Reymond, M. C., et al. (2012). Improved sensorimotor 
performance via stochastic resonance. J. Neurosci. 32, 12612-12618. doi: 10.1523/JNEUROSCI.0680-12.2012

Miranda, D. L., Hsu, W. H., Gravelle, D. C., Petersen, K., Ryzman, R., Niemi, J., et al. (2016). Sensory enhancing insoles improve athletic performance during a hexagonal agility task. J. Biomech. 49, 1058-1063. doi: 10.1016/j.jbiomech.2016.02.022

Priplata, A., Niemi, J., Salen, M., Harry, J., Lipsitz, L. A., and Collins, J. J. (2002). Noise-enhanced human balance control. Phys. Rev. Lett. 89:238101. doi: 10.1103/PhysRevLett.89.238101

Priplata, A. A., Patritti, B. L., Niemi, J. B., Hughes, R., Gravelle, D. C., Lipsitz, L. A., et al. (2006). Noise-enhanced balance control in patients with diabetes and patients with stroke. Ann. Neurol. 59, 4-12. doi: 10.1002/ana.20670

Ross, S. E., and Guskiewicz, K. M. (2006). Effect of coordination training with and without stochastic resonance stimulation on dynamic postural stability of subjects with functional ankle instability and subjects with stable ankles. Clin. J. Sport Med. 16, 323-328. doi: 10.1097/00042752-20060700000007

Scheidt, R. A., Conditt, M. A., Secco, E. L., and Mussa-Ivaldi, F. A. (2005). Interaction of visual and proprioceptive feedback during adaptation of human reaching movements. J. Neurophysiol. 93, 3200-3213. doi: $10.1152 /$ jn. 00947.2004

Severini, G., and Delahunt, E. (2018). Effect of noise stimulation below and above sensory threshold on postural sway during a mildly challenging balance task. Gait Posture 63, 27-32. doi: 10.1016/j.gaitpost.2018.04.031

Shabbott, B. A., and Sainburg, R. L. (2010). Learning a visuomotor rotation: simultaneous visual and proprioceptive information is crucial for visuomotor remapping. Exp. Brain Res. 203, 75-87. doi: 10.1007/s00221-010-2209-3
Shadmehr, R. (2017). Distinct neural circuits for control of movement vs. holding still. J. Neurophysiol. 117, 1431-1460. doi: 10.1152/jn.00840.2016

Shadmehr, R., and Wise, S. P. (2005). The Computational Neurobiology of Reaching and Pointing: A Foundation for Motor Learning. Cambridge, MA: MIT Press.

Sober, S. J., and Sabes, P. N. (2003). Multisensory integration during motor planning. J. Neurosci. 23, 6982-6992. doi: 10.1523/JNEUROSCI.23-18-06982.2003

Sober, S. J., and Sabes, P. N. (2005). Flexible strategies for sensory integration during motor planning. Nat. Neurosci. 8, 490-497. doi: 10.1038/nn1427

van Beers, R. J., Baraduc, P., and Wolpert, D. M. (2002). Role of uncertainty in sensorimotor control. Philos. Trans. R Soc. Lond. B Biol. Sci. 357, 1137-1145. doi: 10.1098/rstb.2002.1101

Vidoni, E. D., and Boyd, L. A. (2009). Preserved motor learning after stroke is related to the degree of proprioceptive deficit. Behav. Brain Funct. 5:36. doi: $10.1186 / 1744-9081-5-36$

Conflict of Interest: The authors declare that the research was conducted in the absence of any commercial or financial relationships that could be construed as a potential conflict of interest.

Copyright $\odot 2019$ Castronovo, Giles Doran, Holden and Severini. This is an openaccess article distributed under the terms of the Creative Commons Attribution License (CC BY). The use, distribution or reproduction in other forums is permitted, provided the original author(s) and the copyright owner(s) are credited and that the original publication in this journal is cited, in accordance with accepted academic practice. No use, distribution or reproduction is permitted which does not comply with these terms. 